\section{AGO erneuert Empfehlung für Capeci- tabin beim metastasierten Brustkrebs}

In ihren aktuellen Empfehlungen spricht sich die Arbeitsgemeinschaft für Gynäkologische Onkologie (AGO) Organkommission «Mamma» für den Einsatz von Capecitabin (Xeloda ${ }^{\circledR}$ ) beim metastasierten Mammakarzinom aus. Damit erneuert die Kommission ihre höchste Empfehlung für die Capecitabin-Monotherapie (www.ago-online.org).

Die AGO empfiehlt aufgrund der überzeugenden Wirksamkeit in ihren aktuellen Leitlinien die Capecitabin-Monotherapie nach Anthrazyklin-Vorbehandlung bei metastasiertem Brustkrebs. Keine andere Substanz hat bei mit Anthrazyklinen vorbehandelten Patientinnen konsistent eine so hohe Wirksamkeit gezeigt [1]. Daher vergibt die AGO auch den höchsten Empfehlungsgrad (++).
Dieser klinische Empfehlungsgrad der AGO besagt konkret, dass die empfohlene Therapie für die Patientinnen von großem Vorteil ist, uneingeschränkt empfohlen wird und durchgeführt werden sollte.

Zweimal die höchste Empfehlung für $\mathrm{Ca}$ pecitabin-Monotherapie

Diese höchste Empfehlung (++) gibt die AGO ebenfalls der Therapie mit Capecitabin nach Taxan- und Anthrazyklin-Vorbehandlung bei fortgeschrittenem Brustkrebs. Damit bleibt Capecitabin auch in diesem Jahr das einzige zugelassene Produkt nach Anthrazyklin- und Taxan-Vorbehandlung, für das diese hohe Empfehlung abgegeben wurde. Durch die guten Ergebnisse gilt die Capecitabin-Gabe hier als Referenztherapie: Bei guter Verträglichkeit verlängert es die mediane Überlebenszeit auf über 15 Monate [2].
Tumorspezifischer Wirkmechanismus schont gesunde Zellen

Da Capecitabin erst vorrangig in Tumorzellen in seine aktive Form umgewandelt wird, werden gesunde Zellen wie etwa Haarzellen geschont. Die Therapie ist damit besonders gut verträglich.

Quellen

1 Miles D, et al:. Clin Breast Cancer 2005; 5:273-278.

2 Fumoleau P, et al.: Eur J Cancer 2004; 40:536-542.

Roche Pharma AG

Öffentlichkeitsarbeit Pharma

Dr. H.-U. Jelitto

Emil-Barell-Straße 1

79639 Grenzach-Wyhlen

Tel. +49 7624 14-3715, Fax -3366

www.roche.de

\section{Therapietreue im Fokus}

Mit der Aufnahme der 2.000sten Patientin hat das PACT-Programm (Patient's Anastrozol Compliance to Therapy) eine wichtige Schwelle überschritten. Das im Oktober 2006 gestartete Projekt untersucht die Bedeutung der Therapietreue in der adjuvanten antihormonellen Brustkrebstherapie mit einem Aromatasehemmer. PACT soll zeigen, ob die Therapie-Abbruchrate durch verbesserte Aufklärung und Motivation der Patientinnen verringert werden kann und welchen Einfluss die Therapietreue auf die Wirksamkeit der adjuvanten Antihormontherapie hat. PACT ist in Deutschland das erste und bislang einzige Kooperationsprojekt zwischen Brustzentren und niedergelassenen Gynäkologen im Bereich der Compliance-Forschung.
Knapp 5.000 postmenopausale Frauen, bei denen ein hormonsensitives Mammakarzinom entfernt wurde, können über einen Zeitraum von maximal 5 Jahren am Programm teilnehmen. Alle Teilnehmerinnen erhalten alle notwendigen Untersuchungen und $\mathrm{Be}$ handlungen entsprechend den aktuellen Standards. Die Hälfte der Frauen bekommt zusätzlich neunmal innerhalb des ersten Therapiejahres von anderen Brustkrebs-Patientinnen erstellte schriftliche Informationen zu ihrer Antihormontherapie und zur Brustkrebserkrankung. Im Rahmen einer sensiblen Gesprächsführung über die adjuvante Therapie wird die Patientin vom Arzt umfassend informiert und in die Therapieentscheidung mit einbezogen.

Nach maximal 5 Jahren werden die Daten ausgewertet und mit Rezeptdaten sowie einer Einschätzung des behandelnden Arztes verglichen. Vor allem wird ausgewertet, ob die zusätzlichen Informationsmaterialien eine höhere Therapietreue bewirkt haben. Die Therapietreue wird mit Hilfe von Fragebögen erfasst, die von der Patientin selbst zu verschiedenen festgelegten Zeitpunkten ausgefüllt werden. Durch die ausführliche Aufklärung und das Ausfüllen der Fragebögen fühlen sich die Patientinnen besonders gut betreut, vor allem die, die zusätzliche Informationen erhalten.

Weitere Informationen bei

AstraZeneca $\mathrm{GmbH}$

Peter Schiffer

Tinsdaler Weg 183, 22880 Wedel

Tel. +49 4103 708-3663

peter.schiffer@astrazeneca.com

\title{
PharmaTicker+++ PharmaTicker+++ PharmaTicker+++ PharmaTicker+++
}

\author{
Lilly Deutschland GmbH. Der Lilly Quality of \\ Life-Preis 2007 wurde im Februar 2008 an drei \\ Wissenschaftler verliehen: Prof. Dr. Dieter Naber \\ und PD Dr. Martin Lambert von der Klinik und \\ Poliklinik des Universitätsklinikums Hamburg-Ep- \\ pendorf sowie PD Dr. Volker Arndt vom Deut- \\ schen Krebsforschungszentrum in Heidelberg \\ erhielten den mit 10.000 Euro dotierten Preis zur \\ Lebensqualitätsforschung. \\ 3K Agentur für Kommunikation $\mathrm{GmbH}$ \\ Bettina Baur \\ Wiesenau 36 \\ 60323 Frankfurt \\ Tel. +49 69 971711-0, Fax -22 \\ info@3k-komm.de
}

essex pharma GmbH. Die Prophylaxe mit NOXAFIL ${ }^{\circledR}$ (Posaconazol) kann nicht nur die pilzbedingte Sterblichkeit verringern, sondern möglicherweise auch Kosten einsparen. Dies bestätigten die Referenten eines Symposiums der essex pharma $\mathrm{GmbH}$ auf dem 28. Deutschen Krebskongress in Berlin. Budgetverantwortliche in der Klinik sollten beachten, dass Posaconazol erstmals seit diesem Jahr im Rahmen des bepreisten Zusatzentgelts abgerechnet werden kann.

Dr. Michael Bonk

Hansaring 97.50670 Köln

Tel. +49 $221912719-27$, Fax -57

Michael.Bonk@publicis-vital-pr.de medac GmbH. Seit 1. April 2008 ist Oncofolic ${ }^{\circledR}$ (Wirkstoff: Natriumfolinat) auch in der Wirkstärke 400 mg erhältlich. Oncofolic ${ }^{\circledR}$ ist in Kombination mit 5-Fluorouracil im Rahmen einer zytotoxischen Behandlung zugelassen. Oncofolic ${ }^{\circledR} 50 \mathrm{mg} / \mathrm{ml}$ Injektions- und Infusionslösung wird als $100 \mathrm{mg}$ (PZN 2406657), 200 mg (PZN 2406686), 300 mg (PZN 2406700) 500 mg (PZN 2406775), 900 mg (PZN 2406835) und jetzt auch als 400 mg Lösung (PZN 4373896) angeboten.

medac $\mathrm{GmbH}$

Dr. Christoph Erdmann

Theaterstraße 6, 22880 Wedel

Tel. +49 4103 8006-404

c.erdmann@medac.de 